\title{
CHANGING PATTERN OF MORTALITY IN ENGLAND AND WALES \\ III. MORTALITY IN OLD AGE
}

\author{
(a) Picture Disclosed by Annual Specific Rates \\ BY \\ WALLIS TAYLOR* AND MARJORIE DAUNCEY \\ Department of Medical Statistics, Birmingham University
}

Previous contributions to this series have dealt with infant mortality and with maternal mortality (Taylor, 1954; Taylor and Dauncey, 1954).

The aim of this survey is to examine what ages are of special interest vis $\dot{a}$ vis the conservation of life, and to complete it we have still to deal with old age. An appropriate definition of what we mean by the term old age in this context will emerge later. It will clear the ground for subsequent discussion if we first ask how many people reach extreme age.

The current Life Table presented in Table $I$ is based on data given in the Registrar General's one per cent. sample of the 1951 census. It sums up present day mortality for a fictitious population of 100,000 males and 100,000 females born in
1952, each subject to the average mortality rate of 1950-52. Of these approximately 90,000 males and 93,000 females would reach age 45, 52,000 males and 68,000 females would reach age 70 , and 8,000 males and 18,000 females would reach age 85. These estimates are based on death rates at the present time. We shall later attempt to show the most probable effects of future changes in mortality. In the 20 years since 1931 the population over 80 years of age in England and Wales has approximately doubled-from 208,000 to 406,000 for females and from 114,000 to 221,000 for males; but very little of this change is due to reduced mortality at high ages. Nearly all the expansion is attributable to the particular age structure of the community,

TABLE I

LIFE TABLES

\begin{tabular}{|c|c|c|c|c|c|c|c|c|}
\hline \multirow{3}{*}{$\frac{\frac{\text { Table } \cdots}{\text { Sex } \cdots}}{\text { Age (yrs) }}$} & \multicolumn{4}{|c|}{ English No. 10 (1930-32) } & \multicolumn{4}{|c|}{ Abridged England and Wales, 1950-52 } \\
\hline & \multicolumn{2}{|c|}{ Male } & \multicolumn{2}{|c|}{ Female } & \multicolumn{2}{|c|}{ Male } & \multicolumn{2}{|c|}{ Female } \\
\hline & $1 x$ Survivors & $\begin{array}{l}o \\
e\end{array}$ & $1 x$ Survivors & $\stackrel{0}{x}^{0}$ & $1 x$ Survivors & $\stackrel{0}{e}^{x}$ & $1 x$ Survivors & $\stackrel{\boldsymbol{e}}{\boldsymbol{e}}^{\boldsymbol{x}}$ \\
\hline $\begin{array}{r}0 \\
1 \\
2 \\
3 \\
4 \\
5 \\
10 \\
15 \\
20 \\
25 \\
30 \\
35 \\
40 \\
45 \\
50 \\
55 \\
60 \\
65 \\
70 \\
75 \\
80 \\
85 \\
90 \\
95 \\
100 \\
105\end{array}$ & $\begin{array}{r}100,000 \\
92,814 \\
91,394 \\
90,794 \\
90,394 \\
90,069 \\
89,023 \\
88,360 \\
87,245 \\
85,824 \\
84,416 \\
82,885 \\
80,935 \\
78,357 \\
74,794 \\
70,041 \\
63,620 \\
54,899 \\
43,361 \\
29,665 \\
16,199 \\
6,377 \\
1,609 \\
232 \\
15 \\
-\end{array}$ & $\begin{array}{c}58 \cdot 74 \\
62 \cdot 25 \\
62 \cdot 21 \\
61 \cdot 62 \\
60 \cdot 89 \\
60 \cdot 11 \\
55 \cdot 79 \\
51 \cdot 19 \\
46 \cdot 81 \\
42 \cdot 54 \\
38 \cdot 21 \\
33 \cdot 87 \\
29 \cdot 62 \\
25 \cdot 51 \\
21 \cdot 60 \\
17 \cdot 89 \\
14 \cdot 43 \\
11 \cdot 30 \\
8 \cdot 62 \\
6 \cdot 43 \\
4 \cdot 74 \\
3 \cdot 50 \\
2 \cdot 63 \\
1 \cdot 97 \\
1 \cdot 48 \\
-\end{array}$ & $\begin{array}{r}100,000 \\
94,545 \\
93,273 \\
92,711 \\
92,334 \\
92,024 \\
91,082 \\
90,420 \\
89,383 \\
88,133 \\
86,792 \\
85,353 \\
83,690 \\
81,660 \\
78,958 \\
75,290 \\
70,204 \\
63,046 \\
53,144 \\
40,040 \\
24,869 \\
11,594 \\
3,611 \\
681 \\
64 \\
2\end{array}$ & $\begin{array}{l}62 \cdot 88 \\
65 \cdot 48 \\
65 \cdot 37 \\
64 \cdot 76 \\
64 \cdot 03 \\
63 \cdot 24 \\
58 \cdot 87 \\
54 \cdot 28 \\
49 \cdot 88 \\
45 \cdot 55 \\
41 \cdot 22 \\
36 \cdot 87 \\
32 \cdot 55 \\
28 \cdot 30 \\
24 \cdot 18 \\
20 \cdot 23 \\
16 \cdot 50 \\
13 \cdot 07 \\
10 \cdot 02 \\
7 \cdot 45 \\
5 \cdot 46 \\
4 \cdot 00 \\
2 \cdot 98 \\
2 \cdot 22 \\
1 \cdot 65 \\
1 \cdot 22\end{array}$ & $\begin{array}{r}100,000 \\
96,725 \\
96,488 \\
96,353 \\
96,255 \\
96,180 \\
95,799 \\
95,541 \\
95,089 \\
94,436 \\
93,745 \\
92,934 \\
91,885 \\
90,319 \\
87,540 \\
82,960 \\
75,852 \\
65,599 \\
52,324 \\
36,718 \\
21,042 \\
8,062 \\
1,903 \\
238 \\
- \\
-\end{array}$ & $\begin{array}{c}66 \cdot 33 \\
67 \cdot 57 \\
66 \cdot 74 \\
65 \cdot 83 \\
64 \cdot 90 \\
63 \cdot 95 \\
59 \cdot 19 \\
54 \cdot 34 \\
49 \cdot 59 \\
44 \cdot 92 \\
40 \cdot 32 \\
35 \cdot 56 \\
30 \cdot 94 \\
26 \cdot 43 \\
22 \cdot 19 \\
18 \cdot 27 \\
14 \cdot 75 \\
11 \cdot 67 \\
8 \cdot 99 \\
6 \cdot 75 \\
4 \cdot 92 \\
3 \cdot 83 \\
3 \cdot 12 \\
2 \cdot 50 \\
- \\
-\end{array}$ & $\begin{array}{c}100,000 \\
97,478 \\
97,266 \\
97,153 \\
97,072 \\
97,011 \\
96,782 \\
96,603 \\
96,291 \\
95,854 \\
95,296 \\
94,624 \\
93,764 \\
92,544 \\
90,636 \\
87,852 \\
83,670 \\
\mathbf{7 7 , 3 5 0} \\
\mathbf{6 7 , 7 9 2} \\
\mathbf{5 4 , 1 9 0} \\
\mathbf{3 5 , 9 5 3} \\
17,731 \\
\mathbf{5 , 4 8 9} \\
988 \\
99 \\
-\end{array}$ & $\begin{array}{c}71 \cdot 47 \\
72 \cdot 31 \\
71 \cdot 47 \\
70 \cdot 55 \\
69 \cdot 61 \\
68 \cdot 65 \\
63 \cdot 81 \\
58 \cdot 92 \\
54 \cdot 11 \\
49 \cdot 34 \\
44 \cdot 62 \\
39 \cdot 91 \\
35 \cdot 26 \\
30 \cdot 69 \\
26 \cdot 28 \\
22 \cdot 04 \\
18 \cdot 01 \\
14 \cdot 28 \\
10.94 \\
8.06 \\
5 \cdot 88 \\
4 \cdot 35 \\
3.50 \\
3.00 \\
2.50 \\
-\end{array}$ \\
\hline
\end{tabular}

* University Research Fellow supported by the Rockefeller Foundation. 
whereby each year the number of entrants into the highest aged groups has been successively larger.

Over the period of our inquiry (1931-50) mortality has uniformly decreased, and in some age groups it has decreased very rapidly. Our first question is: has mortality decreased in the older age group? The period $1931-51$ is unique in that the only firm estimates of the size of the population are 20 years apart. (The count of the population in 1939 was probably less accurate than a census sensu stricto). As is inevitable some error has crept into the annual population figures supplied by the Registrar-General, which are compiled by subtracting deaths and adding new entries into each age group. A check based on the Census population shows this to be true. Since the population estimated in the Census year is certainly more exact than the population estimated in the preceding year, this has been accepted, and graphical estimates of the population have been made for the two years adjoining 1951. The difference in the death rates is appreciable.

\begin{tabular}{l|c|c|c|c|c|c}
\hline $\begin{array}{c}\text { Age } \\
\text { Group } \\
\text { 80 and } \\
\text { Over }\end{array}$ & Year & $\begin{array}{c}\text { Registrar- } \\
\text { General's } \\
\text { Estimated } \\
\text { Popu- } \\
\text { lation }\end{array}$ & Deaths & $\begin{array}{c}\text { Registrar- } \\
\text { General's } \\
\text { Death } \\
\text { Rate } \\
-\end{array}$ & $\begin{array}{c}\text { Estimated } \\
\text { Correc- } \\
\text { tion }\end{array}$ & $\begin{array}{c}\text { Cor- } \\
\text { rected } \\
\text { Rate }\end{array}$ \\
\hline Males & 1950 & 237,000 & 44,373 & $187 \cdot 2$ & 215,000 & $206 \cdot 4$ \\
& 1951 & 229,000 & 49,275 & $215 \cdot 2$ & 220,900 & $223 \cdot 1$ \\
Females & 1952 & 240,000 & 45,280 & $188 \cdot 7$ & 226,000 & 200.4 \\
\hline & 1950 & 423,000 & 67,490 & $159 \cdot 6$ & 395,000 & $170 \cdot 9$ \\
& 1952 & 407,000 & 75,432 & $185 \cdot 3$ & 405,900 & $185 \cdot 8$ \\
\hline
\end{tabular}

These reservations, must therefore be borne in mind when an attempt is made to calculate death rates from the published population. Table II gives as moving averages the annual age-specific death rates referable to each sex for the period under discussion. They show that:

(1) During the period 1931-53, there has been a decline in the specific death rates for females in each quinquennium from 55 years of age up to the terminal age group of 80 years and over; but this fall has not been sustained in the sense defined in the first communication of this series, since there are small peaks in the period 1944-53;

(2) The same is true of males with two reservations:

(a) the proportionate overall decline has been less;

(b) there has been no appreciable drop in the quinquennium 60-64.

For the highest age groups our picture is undoubtedly biased by the inaccuracy of annual population estimates. There seems to be evidence ( $c f$. Registrar-General's Census Volumes) that population estimates at the end of the period were commonly too high for the old-age groups. So the initial fall and eventual slight rise in the death rates may be to some extent an artefact. This consideration prompts us to compare the Life Tables for the years 1950-52 with similar Tables for 1930-32. This tells us nothing about changes in the intervening period, it does enable us to compare the two terminal triennia referable to census populations; but we cannot yet attain very great accuracy with respect to the latest Census since the only age and sex breakdown so far available is referable to a one per cent. sample. With this reservation we must scrutinize Table I exhibiting an abridged Life Table based on these values.

The difficulty of measuring small survival changes for Scotland in the highest age groups is exemplified in the 1950-52 Life Tables prepared by the Government Actuary, who comments:

To some extent, therefore, the figures (for Scotland) may be affected by short-term fluctuations from the general secular trend due to the incidence of epidemics or adverse meteorological conditions; at the older ages

TABLE II

TOTAL DEATH RATE

Five-Year Moving Average

\begin{tabular}{|c|c|c|c|c|c|c|c|c|c|c|c|c|c|c|c|c|c|c|c|c|}
\hline Sex & Age & $\begin{array}{c}1931- \\
1935\end{array}$ & $\begin{array}{c}1932- \\
1936\end{array}$ & $\begin{array}{l}1933- \\
1937\end{array}$ & $\begin{array}{l}1934 \\
1938\end{array}$ & $\begin{array}{c}1935 \\
1939 \\
\end{array}$ & $\begin{array}{l}1936- \\
1940\end{array}$ & $\begin{array}{l}1937- \\
1941\end{array}$ & $\begin{array}{r}1938- \\
1942\end{array}$ & $\begin{array}{c}1939 \\
1943\end{array}$ & $\begin{array}{r}1940- \\
1944\end{array}$ & $\begin{array}{l}1941- \\
1945\end{array}$ & $\begin{array}{l}1942- \\
1946\end{array}$ & $\begin{array}{c}1943 \\
1947\end{array}$ & $\begin{array}{l}1944 \\
1948\end{array}$ & $\begin{array}{c}1945 \\
1949\end{array}$ & $\begin{array}{c}1946- \\
1950\end{array}$ & $\begin{array}{l}1947- \\
1951\end{array}$ & $\begin{array}{c}1948- \\
1952\end{array}$ & $-1949-$ \\
\hline Male & $\begin{array}{r}50-54 \\
55-59 \\
60-64 \\
65-69 \\
70-74 \\
75-79 \\
80 \text { and } \\
\text { over }\end{array}$ & $\begin{array}{r}13.2 \\
19.3 \\
28.8 \\
45.9 \\
73.3 \\
117.7 \\
204.5\end{array}$ & $\begin{array}{r}13 \cdot 1 \\
19 \cdot 2 \\
28 \cdot 9 \\
45 \cdot 3 \\
73 \cdot 1 \\
117 \cdot 7 \\
203 \cdot 4\end{array}$ & $\begin{array}{r}13 \cdot 3 \\
19 \cdot 4 \\
29 \cdot 3 \\
45 \cdot 1 \\
72 \cdot 9 \\
117 \cdot 6 \\
203 \cdot 8\end{array}$ & \begin{tabular}{|}
$13 \cdot 1$ \\
$19 \cdot 1$ \\
$29 \cdot 3$ \\
$44 \cdot 6$ \\
$72 \cdot 2$ \\
$116 \cdot 1$ \\
$200 \cdot 5$
\end{tabular} & \begin{tabular}{|c|}
$13 \cdot 0$ \\
$19 \cdot 2$ \\
$29 \cdot 6$ \\
$44 \cdot 6$ \\
$72 \cdot 6$ \\
$117 \cdot 6$ \\
$204 \cdot 4$
\end{tabular} & \begin{tabular}{|r|}
$13 \cdot 4$ \\
$20 \cdot 1$ \\
$30 \cdot 7$ \\
$45 \cdot 8$ \\
$73 \cdot 4$ \\
$120 \cdot 8$ \\
$210 \cdot 7$
\end{tabular} & $\begin{array}{r}13.4 \\
20.3 \\
30.8 \\
46.0 \\
72.4 \\
119.9 \\
209.6\end{array}$ & \begin{tabular}{|c|}
$13 \cdot 0$ \\
$19 \cdot 9$ \\
$30 \cdot 1$ \\
$45 \cdot 3$ \\
$70 \cdot 3$ \\
$116 \cdot 8$ \\
$203 \cdot 1$ \\
$203 \cdot 1$ \\
\end{tabular} & \begin{tabular}{|c|}
$12 \cdot 8$ \\
$19 \cdot 9$ \\
$29 \cdot 9$ \\
$45 \cdot 1$ \\
$69 \cdot 2$ \\
$115 \cdot 5$ \\
$200 \cdot 5$
\end{tabular} & \begin{tabular}{|r|r}
12.5 \\
19.6 \\
29.5 \\
44.6 \\
67.4 \\
111.2 \\
192.9
\end{tabular} & $\begin{array}{r}11 \cdot 7 \\
18 \cdot 5 \\
28 \cdot 2 \\
42 \cdot 8 \\
64 \cdot 4 \\
104 \cdot 8 \\
181 \cdot 4\end{array}$ & 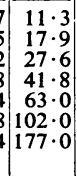 & \begin{tabular}{|r|}
$11 \cdot 3$ \\
$17 \cdot 8$ \\
$27 \cdot 8$ \\
$41 \cdot 7$ \\
$63 \cdot 6$ \\
$102 \cdot 4$ \\
$180 \cdot 5$
\end{tabular} & $\begin{array}{r}10 \cdot 9 \\
17 \cdot 5 \\
27 \cdot 7 \\
41 \cdot 2 \\
63 \cdot 0 \\
100 \cdot 0 \\
176 \cdot 2\end{array}$ & $\begin{array}{r}10.8 \\
17.4 \\
27.8 \\
41.4 \\
63.8 \\
100.7 \\
178.7\end{array}$ & \begin{tabular}{|r|}
10.7 \\
17.4 \\
28.0 \\
41.8 \\
64.6 \\
101.9 \\
$18 ! .9$
\end{tabular} & $\begin{array}{r}10 \cdot 7 \\
17 \cdot 6 \\
28 \cdot 6 \\
43 \cdot 3 \\
66 \cdot 9 \\
105 \cdot 1 \\
189 \cdot 2\end{array}$ & \begin{tabular}{|r}
$10 \cdot 6$ \\
$17 \cdot 5$ \\
$28 \cdot 4$ \\
$43 \cdot 5$ \\
$66 \cdot 8$ \\
$104 \cdot 8$ \\
$187 \cdot 6$
\end{tabular} & \begin{tabular}{|r|r}
$10 \cdot 6$ \\
$17 \cdot 6$ \\
$28 \cdot 6$ \\
$44 \cdot 6$ \\
$68 \cdot 1$ \\
$107 \cdot 3$ \\
$193 \cdot 0$
\end{tabular} \\
\hline Female & $\begin{array}{r}50-54 \\
55-59 \\
60-64 \\
65-69 \\
70-74 \\
75-79 \\
80 \text { and } \\
\text { over }\end{array}$ & $\begin{array}{r}9 \cdot 4 \\
13.6 \\
21 \cdot 1 \\
33.5 \\
55 \cdot 8 \\
91.3 \\
177.1\end{array}$ & $\begin{array}{r}9.2 \\
13.4 \\
20.9 \\
33.2 \\
55.2 \\
90.1 \\
174.6\end{array}$ & $\begin{array}{r}9 \cdot 2 \\
13 \cdot 2 \\
20 \cdot 9 \\
33 \cdot 0 \\
55 \cdot 1 \\
89 \cdot 7 \\
173 \cdot 8\end{array}$ & \begin{tabular}{|r}
8.9 \\
12.8 \\
20.5 \\
32.4 \\
53.6 \\
87.8 \\
167.9
\end{tabular} & \begin{tabular}{|r|r|}
$8 \cdot 8$ \\
$12 \cdot 7$ \\
$20 \cdot 4$ \\
$32 \cdot 2$ \\
$53 \cdot 2$ \\
$88 \cdot 4$ \\
$169 \cdot 9$
\end{tabular} & \begin{tabular}{|r|}
$8 \cdot 9$ \\
$12 \cdot 9$ \\
$20 \cdot 7$ \\
$32 \cdot 8$ \\
$54 \cdot 2$ \\
$90 \cdot 4$ \\
$175 \cdot 5$
\end{tabular} & $\begin{array}{r}8.8 \\
12.7 \\
20.3 \\
32.4 \\
53.5 \\
89.4 \\
173.4\end{array}$ & \begin{tabular}{|r|}
$8 \cdot 5$ \\
$12 \cdot 4$ \\
$19 \cdot 4$ \\
$31 \cdot 3$ \\
$51 \cdot 6$ \\
$86 \cdot 5$ \\
$167 \cdot 3$
\end{tabular} & \begin{tabular}{|r|}
$8 \cdot 3$ \\
12.2 \\
18.9 \\
30.9 \\
$51 \cdot 0$ \\
85.5 \\
$167 \cdot 2$
\end{tabular} & \begin{tabular}{|r|}
$8 \cdot 1$ \\
$11 \cdot 8$ \\
$18 \cdot 3$ \\
$29 \cdot 9$ \\
$49 \cdot 4$ \\
$82 \cdot 3$ \\
$161 \cdot 2$
\end{tabular} & $\begin{array}{r}7 \cdot 6 \\
11 \cdot 1 \\
17 \cdot 2 \\
28 \cdot 2 \\
46 \cdot 8 \\
77 \cdot 7 \\
152 \cdot 4\end{array}$ & \begin{tabular}{|r|r|}
$6 \cdot 3$ \\
$10 \cdot 7$ \\
$16 \cdot 7$ \\
$27 \cdot 2$ \\
8 \\
7 & $75 \cdot 7$ \\
4 & $76 \cdot 0$ \\
& $149 \cdot 2$ \\
\end{tabular} & \begin{tabular}{|r}
$7 \cdot 2$ \\
$10 \cdot 5$ \\
$16 \cdot 6$ \\
$26 \cdot 9$ \\
$45 \cdot 9$ \\
$76 \cdot 7$ \\
$151 \cdot 5$
\end{tabular} & $\begin{array}{r}6 \cdot 9 \\
10 \cdot 2 \\
16 \cdot 2 \\
26 \cdot 1 \\
44 \cdot 9 \\
74 \cdot 9 \\
147 \cdot 3\end{array}$ & $\begin{array}{r}6 \cdot 8 \\
10 \cdot 1 \\
16 \cdot 1 \\
26 \cdot 0 \\
45 \cdot 3 \\
76 \cdot 0 \\
150 \cdot 1\end{array}$ & $\begin{array}{r}6 \cdot 6 \\
10.0 \\
16.0 \\
26.0 \\
45.3 \\
76.9 \\
153 \cdot 1\end{array}$ & $\begin{array}{r}6.6 \\
9.9 \\
15.9 \\
26.4 \\
45.8 \\
78 \cdot 5 \\
159.7\end{array}$ & \begin{tabular}{|r|r}
6.4 \\
9.7 \\
15.6 \\
\\
26.0 \\
54.8 \\
77.2 \\
7 & 159.5
\end{tabular} & $\begin{array}{r}6 \cdot 2 \\
9.6 \\
15 \cdot 5 \\
26 \cdot 1 \\
44 \cdot 7 \\
77 \cdot 9 \\
164 \cdot 4\end{array}$ \\
\hline
\end{tabular}


in particular the death rate from respiratory and cardiac diseases can vary considerably from one year to another according to the nature of the weather in the winter months. Nevertheless, it is not to be supposed that if the comparison has been based on the experience of periods other than those used in the construction of Table $B$ the broad picture of secular trend which the table is intended to provide would have differed in any important respect (Government Actuary, 1954).

At the end of the 19th century there had already been a remarkable increase in the expectation of life at birth without as yet any clear indication that the risk of death in the first year of life had begun to lessen. Throughout the first half of the 20th century there has been a sustained and spectacular decline in infant mortality, which so far shows no immediate signs of abatement. Till far into the first half of the 20th century there was no indication of a reduction in mortality at the latter end of life, at or above the 60-year level. With due regard to the sources of error mentioned, it now seems that we have entered a new phase characterized by declining mortality in the terminal age groups. How far this process may continue will be discussed later.

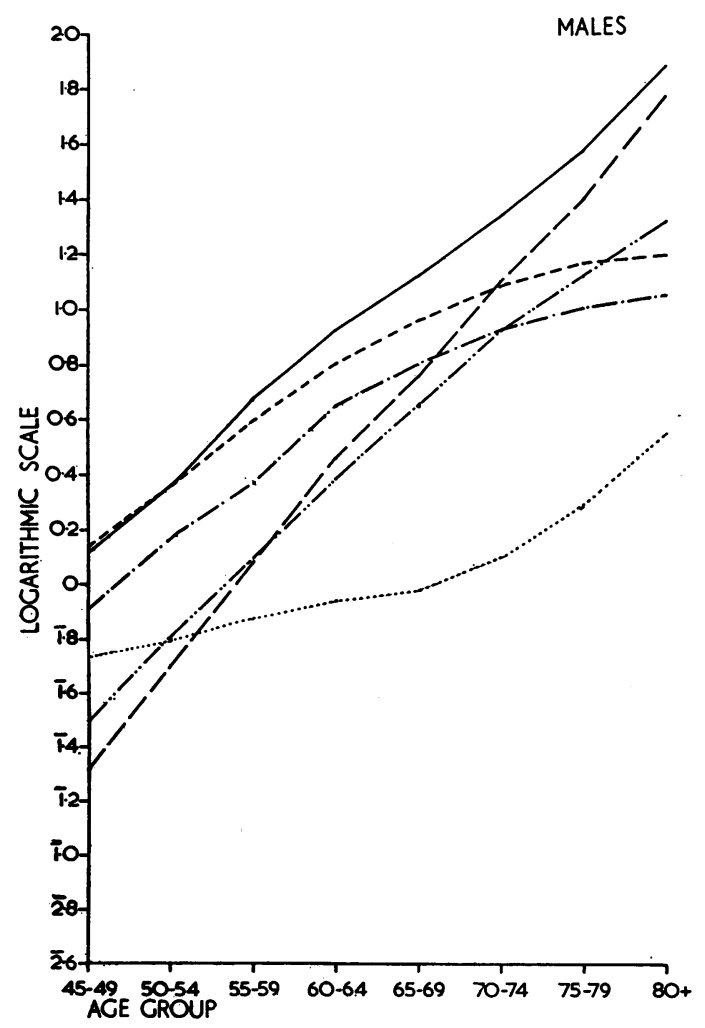

\section{Specific Causes of Death}

The incidence of assigned specific causes of death may be scrutinized in two ways. We may exhibit the percentage contribution to total deaths or the actual rate of any one specific disease. Of itself, the elimination of one cause will automatically affect the percentage rating, but not the ranking, of every other cause of death.

Tables III and IV (opposite) show that the alternative methods of actual rate and percentage contribution exhibit most clearly the interplay of different specific causes of death. The Figure illustrates another method; here the actual specific mortality rates are plotted on semi-log paper for each age group. As in previous communications (Taylor, 1954, Taylor and Dauncey, 1954), the use of semi-log paper discloses a straight line as the criterion of a constant proportionate change. This procedure shows up most clearly the tempo of death assigned to a particular cause as age advances; but it is only by comparison of the figures with the total death-rate that it is possible to decide whether a specific cause of death is becoming more important vis $\grave{a}$ vis all

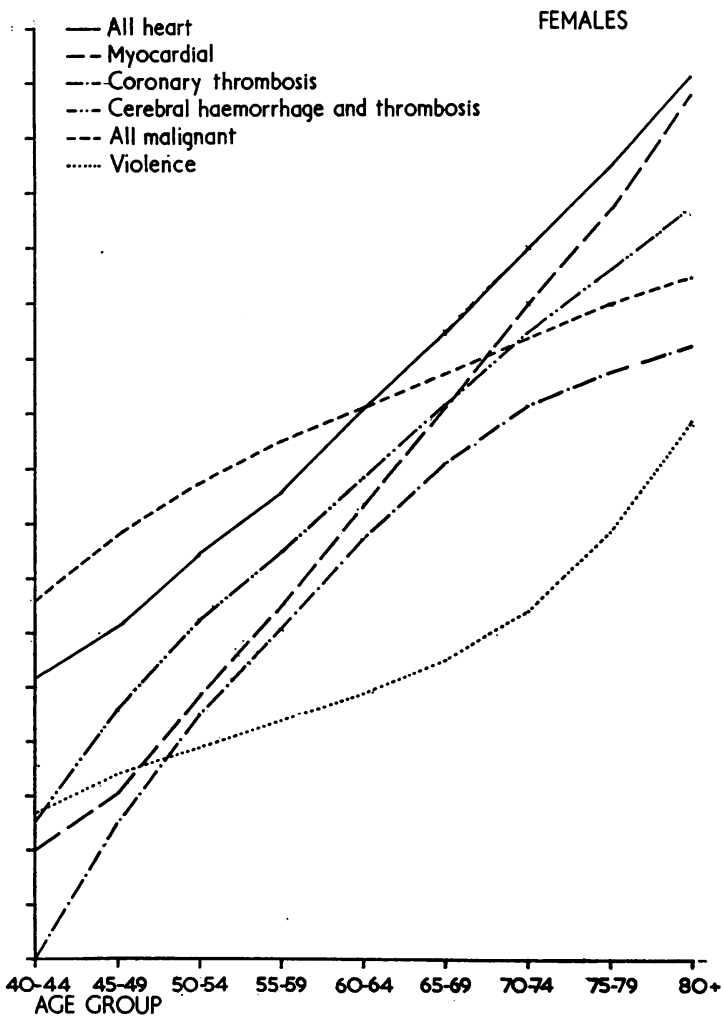

FEMALES 
TABLE III

DEATH RATE PER 1,000 POPULATION (1931 AND 1940)

\begin{tabular}{|c|c|c|c|c|c|c|c|c|c|c|c|c|c|c|c|c|c|c|}
\hline \multicolumn{2}{|l|}{ Sex } & \multicolumn{8}{|c|}{ Males } & \multicolumn{9}{|c|}{ Females } \\
\hline \multirow[b]{2}{*}{ Cause of Death } & \multirow[b]{2}{*}{ Year } & \multicolumn{8}{|c|}{ Age Group } & \multicolumn{9}{|c|}{ Age Group } \\
\hline & & $\begin{array}{c}45- \\
49\end{array}$ & $50-54$ & $55-59$ & $60-64$ & $65-69$ & $\overline{70-74}$ & $75-79$ & $\begin{array}{l}80 \text { and } \\
\text { Over }\end{array}$ & $\begin{array}{c}40- \\
44\end{array}$ & $\begin{array}{c}45- \\
49\end{array}$ & $50-$ & $55-59$ & $60-64$ & $65-69$ & $70-74$ & $\overline{75-79}$ & $\begin{array}{l}80 \text { and } \\
\text { Over }\end{array}$ \\
\hline All Malignant Diseases & $\begin{array}{l}1931 \\
1949\end{array}$ & \begin{tabular}{|l|}
$1 \cdot 19$ \\
$1 \cdot 42$
\end{tabular} & $\begin{array}{l}2 \cdot 10 \\
2 \cdot 45\end{array}$ & \begin{tabular}{|l|}
3.61 \\
3.99 \\
\end{tabular} & $\begin{array}{l}5 \cdot 90 \\
6 \cdot 17\end{array}$ & $\begin{array}{l}9 \cdot 09 \\
8 \cdot 84\end{array}$ & $\begin{array}{l}11.82 \\
11.99\end{array}$ & $\begin{array}{l}13 \cdot 38 \\
14 \cdot 72\end{array}$ & $\begin{array}{l}14.03 \\
15 \cdot 64\end{array}$ & $\begin{array}{r}.99 \\
0.84\end{array}$ & $\begin{array}{l}1 \cdot 70 \\
1 \cdot 50\end{array}$ & $\begin{array}{l}2 \cdot 60 \\
2 \cdot 20\end{array}$ & $\begin{array}{l}3 \cdot 54 \\
3 \cdot 14\end{array}$ & $\begin{array}{l}4 \cdot 82 \\
4 \cdot 16\end{array}$ & $\begin{array}{l}6 \cdot 62 \\
5 \cdot 75\end{array}$ & $\begin{array}{l}8 \cdot 80 \\
7 \cdot 76\end{array}$ & $\begin{array}{l}11 \cdot 14 \\
10 \cdot 05\end{array}$ & $\begin{array}{l}12.83 \\
12.01\end{array}$ \\
\hline $\begin{array}{l}\text { Myocardiac Diseases (1) } \\
\text { Coronary Thrombosis (2) } \\
\text { Other Heart Diseases (3) }\end{array}$ & $\begin{array}{l}1931 \\
1949 \\
1931 \\
1949 \\
1931 \\
1949\end{array}$ & $\begin{array}{l}0 \cdot 51 \\
0 \cdot 22 \\
0 \cdot 17 \\
0 \cdot 82 \\
0 \cdot 65 \\
0 \cdot 32\end{array}$ & $\begin{array}{l}0 \cdot 97 \\
0 \cdot 51 \\
0 \cdot 37 \\
1 \cdot 53 \\
0 \cdot 99 \\
0 \cdot 41\end{array}$ & $\begin{array}{l}1 \cdot 72 \\
1 \cdot 22 \\
0 \cdot 61 \\
2 \cdot 75 \\
1 \cdot 60 \\
0 \cdot 70\end{array}$ & $\begin{array}{l}3 \cdot 55 \\
2 \cdot 98 \\
1 \cdot 01 \\
4 \cdot 49 \\
2 \cdot 76 \\
1 \cdot 05\end{array}$ & $\begin{array}{l}6 \cdot 98 \\
5 \cdot 93 \\
1 \cdot 52 \\
6 \cdot 22 \\
4 \cdot 31 \\
1 \cdot 43\end{array}$ & $\begin{array}{r}13 \cdot 70 \\
12 \cdot 60 \\
2 \cdot 03 \\
8 \cdot 64 \\
6 \cdot 84 \\
2 \cdot 13\end{array}$ & \begin{tabular}{r|}
$24 \cdot 83$ \\
$25 \cdot 32$ \\
$2 \cdot 27$ \\
$10 \cdot 38$ \\
$9 \cdot 66$ \\
$2 \cdot 78$
\end{tabular} & $\begin{array}{r}47 \cdot 74 \\
60 \cdot 48 \\
2 \cdot 25 \\
11 \cdot 21 \\
12 \cdot 59 \\
3 \cdot 53\end{array}$ & $\begin{array}{l}0 \cdot 19 \\
0 \cdot 10 \\
0 \cdot 01 \\
0 \cdot 04 \\
0 \cdot 50 \\
0 \cdot 29\end{array}$ & $\begin{array}{l}0 \cdot 38 \\
0 \cdot 17 \\
0 \cdot 04 \\
0 \cdot 13 \\
0 \cdot 73 \\
0 \cdot 39\end{array}$ & $\begin{array}{l}0 \cdot 67 \\
0 \cdot 38 \\
0 \cdot 06 \\
0 \cdot 31 \\
0 \cdot 91 \\
0 \cdot 52\end{array}$ & $\begin{array}{c}1 \cdot 40 \\
0 \cdot 80 \\
0 \cdot 15 \\
0 \cdot 67 \\
1 \cdot 52 \\
0 \cdot 66\end{array}$ & $\begin{array}{l}2 \cdot 90 \\
1 \cdot 85 \\
0 \cdot 34 \\
1 \cdot 46 \\
2 \cdot 37 \\
0 \cdot 88\end{array}$ & $\begin{array}{l}5 \cdot 59 \\
4 \cdot 20 \\
0 \cdot 56 \\
2 \cdot 73 \\
3 \cdot 87 \\
1 \cdot 25\end{array}$ & $\begin{array}{r}11 \cdot 09 \\
10 \cdot 30 \\
0 \cdot 84 \\
4 \cdot 29 \\
6 \cdot 20 \\
1 \cdot 93\end{array}$ & $\begin{array}{r}20 \cdot 93 \\
22 \cdot 20 \\
1 \cdot 09 \\
5 \cdot 79 \\
9 \cdot 21 \\
2 \cdot 81\end{array}$ & $\begin{array}{r}43.32 \\
56.91 \\
1.37 \\
6.69 \\
12.74 \\
3.90\end{array}$ \\
\hline All Heart Diseases & $\begin{array}{l}1931 \\
1949 \\
\end{array}$ & $\begin{array}{l}1 \cdot 33 \\
1 \cdot 36\end{array}$ & $\begin{array}{l}2 \cdot 33 \\
2 \cdot 45 \\
\end{array}$ & $\begin{array}{l}3 \cdot 93 \\
4 \cdot 67 \\
\end{array}$ & $\begin{array}{l}7 \cdot 32 \\
8 \cdot 52\end{array}$ & $\begin{array}{l}12 \cdot 81 \\
13 \cdot 58\end{array}$ & \begin{tabular}{|l|}
$22 \cdot 57$ \\
$23 \cdot 37$
\end{tabular} & $\begin{array}{l}36 \cdot 76 \\
38 \cdot 48\end{array}$ & $\begin{array}{l}62 \cdot 58 \\
75 \cdot 22\end{array}$ & $\begin{array}{l}0 \cdot 70 \\
0 \cdot 43\end{array}$ & $\overline{1 \cdot 15}$ & $\begin{array}{l}1 \cdot 64 \\
1 \cdot 21\end{array}$ & $\begin{array}{l}3 \cdot 07 \\
2 \cdot 13\end{array}$ & $\begin{array}{l}5 \cdot 61 \\
4 \cdot 19\end{array}$ & $\begin{array}{r}10 \cdot 02 \\
8 \cdot 18\end{array}$ & \begin{tabular}{|l|}
$18 \cdot 13$ \\
$16 \cdot 52$
\end{tabular} & $\overline{31 \cdot 23}$ & $\begin{array}{l}57 \cdot 43 \\
67 \cdot 50\end{array}$ \\
\hline 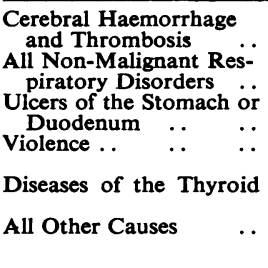 & $\begin{array}{l}1931 \\
1949 \\
1931 \\
1949 \\
1931 \\
1949 \\
1931 \\
1949 \\
1931 \\
1949 \\
1931 \\
1949\end{array}$ & $\begin{array}{l}0 \cdot 24 \\
0 \cdot 31 \\
1 \cdot 37 \\
0 \cdot 81 \\
0 \cdot 37 \\
0 \cdot 25 \\
0 \cdot 88 \\
0 \cdot 55 \\
0 \cdot 02 \\
0 \cdot 01 \\
4 \cdot 25 \\
1 \cdot 84\end{array}$ & $\begin{array}{l}0.46 \\
0.63 \\
1.85 \\
1.58 \\
0.41 \\
0.33 \\
1.00 \\
0.61 \\
0.02 \\
0.02 \\
5.30 \\
2.45\end{array}$ & $\begin{array}{l}0.99 \\
1 \cdot 25 \\
2 \cdot 30 \\
2 \cdot 87 \\
0.46 \\
0.45 \\
1 \cdot 23 \\
0.73 \\
0.03 \\
0.01 \\
6.91 \\
3.51\end{array}$ & $\begin{array}{l}1 \cdot 79 \\
2 \cdot 59 \\
3 \cdot 18 \\
4 \cdot 70 \\
0 \cdot 47 \\
0 \cdot 61 \\
1 \cdot 32 \\
0 \cdot 89 \\
0.03 \\
0.02 \\
9 \cdot 42 \\
5 \cdot 03\end{array}$ & \begin{tabular}{|r|}
$3 \cdot 56$ \\
$4 \cdot 69$ \\
$4 \cdot 90$ \\
$6 \cdot 61$ \\
$0 \cdot 49$ \\
$0 \cdot 70$ \\
$1 \cdot 54$ \\
$0 \cdot 95$ \\
$0 \cdot 04$ \\
0.02 \\
$15 \cdot 31$ \\
$7 \cdot 58$
\end{tabular} & \begin{tabular}{|r|}
$6 \cdot 02$ \\
$8 \cdot 55$ \\
$8 \cdot 14$ \\
$9 \cdot 15$ \\
0.50 \\
0.76 \\
1.78 \\
1.26 \\
0.02 \\
0.02 \\
24.42 \\
11.58
\end{tabular} & $\begin{array}{r}10 \cdot 02 \\
13.54 \\
14.07 \\
13.35 \\
0.51 \\
0.90 \\
2.57 \\
1.95 \\
0.03 \\
0.03 \\
42.55 \\
20 \cdot 28\end{array}$ & $\begin{array}{r}15 \cdot 01 \\
21 \cdot 90 \\
29.48 \\
23 \cdot 17 \\
0.54 \\
0.88 \\
4.39 \\
3.52 \\
0.03 \\
0.01 \\
86.47 \\
44 \cdot 83\end{array}$ & \begin{tabular}{|l|}
$0 \cdot 12$ \\
$0 \cdot 13$ \\
$0 \cdot 44$ \\
$0 \cdot 26$ \\
$0 \cdot 04$ \\
$0 \cdot 02$ \\
$0 \cdot 19$ \\
$0 \cdot 14$ \\
$0 \cdot 09$ \\
$0 \cdot 02$ \\
$2 \cdot 48$ \\
$0 \cdot 99$
\end{tabular} & \begin{tabular}{l|}
$0 \cdot 29$ \\
$0 \cdot 34$ \\
$0 \cdot 52$ \\
$0 \cdot 36$ \\
$0 \cdot 09$ \\
$0 \cdot 04$ \\
$0 \cdot 24$ \\
$0 \cdot 19$ \\
$0 \cdot 13$ \\
$0 \cdot 03$ \\
$2 \cdot 75$ \\
$1 \cdot 18$
\end{tabular} & $\begin{array}{l}0 \cdot 59 \\
0 \cdot 74 \\
0 \cdot 78 \\
0 \cdot 56 \\
0 \cdot 10 \\
0 \cdot 05 \\
0 \cdot 34 \\
0 \cdot 24 \\
0 \cdot 15 \\
0 \cdot 04 \\
3 \cdot 54 \\
1 \cdot 50\end{array}$ & \begin{tabular}{|l|}
0.98 \\
1.29 \\
1.17 \\
0.94 \\
0.13 \\
0.06 \\
0.40 \\
0.30 \\
0.18 \\
0.07 \\
4.73 \\
2.06
\end{tabular} & $\begin{array}{l}1 \cdot 73 \\
2 \cdot 33 \\
1.94 \\
1.63 \\
0 \cdot 13 \\
0 \cdot 13 \\
0.51 \\
0.38 \\
0.20 \\
0.10 \\
7.01 \\
3.05\end{array}$ & $\begin{array}{r}3 \cdot 19 \\
4 \cdot 28 \\
3 \cdot 41 \\
2 \cdot 81 \\
0 \cdot 17 \\
0 \cdot 16 \\
0 \cdot 71 \\
0 \cdot 50 \\
0 \cdot 22 \\
0 \cdot 14 \\
10 \cdot 35 \\
4 \cdot 70\end{array}$ & \begin{tabular}{|}
$5 \cdot 37$ \\
$8 \cdot 10$ \\
$6 \cdot 51$ \\
$5 \cdot 22$ \\
$0 \cdot 17$ \\
$0 \cdot 26$ \\
$1 \cdot 13$ \\
$0 \cdot 77$ \\
$0 \cdot 21$ \\
$0 \cdot 16$ \\
$17 \cdot 13$ \\
$8 \cdot 11$
\end{tabular} & $\begin{array}{r}9 \cdot 05 \\
13 \cdot 76 \\
12 \cdot 15 \\
8 \cdot 71 \\
0 \cdot 23 \\
0 \cdot 31 \\
1.95 \\
1.49 \\
0 \cdot 21 \\
0 \cdot 14 \\
30 \cdot 41 \\
13 \cdot 76\end{array}$ & $\begin{array}{r}14 \cdot 69 \\
21 \cdot 94 \\
26 \cdot 85 \\
18 \cdot 71 \\
0 \cdot 25 \\
0 \cdot 37 \\
4 \cdot 35 \\
3 \cdot 69 \\
0 \cdot 16 \\
0 \cdot 10 \\
69 \cdot 72 \\
33 \cdot 95\end{array}$ \\
\hline Total Death Rate & $\begin{array}{l}1931 \\
1949\end{array}$ & $\begin{array}{l}9 \cdot 65 \\
6 \cdot 55\end{array}$ & $\begin{array}{l}13 \cdot 46 \\
10 \cdot 52\end{array}$ & $\begin{array}{l}19 \cdot 47 \\
17 \cdot 48\end{array}$ & $\begin{array}{l}29 \cdot 43 \\
28 \cdot 53\end{array}$ & $\begin{array}{l}47 \cdot 56 \\
42 \cdot 97\end{array}$ & $\begin{array}{l}75 \cdot 27 \\
66 \cdot 68\end{array}$ & $\begin{array}{l}119 \cdot 89 \\
103 \cdot 25\end{array}$ & $\begin{array}{l}212 \cdot 53 \\
195 \cdot 17\end{array}$ & $\begin{array}{l}5 \cdot 05 \\
2 \cdot 83\end{array}$ & $\begin{array}{l}6 \cdot 87 \\
4 \cdot 33\end{array}$ & $\begin{array}{l}9 \cdot 74 \\
6 \cdot 54\end{array}$ & $\begin{array}{r}14 \cdot 20 \\
9 \cdot 99\end{array}$ & $\begin{array}{l}21 \cdot 95 \\
15 \cdot 97\end{array}$ & $\begin{array}{l}34 \cdot 68 \\
26 \cdot 52\end{array}$ & $\begin{array}{l}57 \cdot 45 \\
46 \cdot 90\end{array}$ & $\begin{array}{l}96 \cdot 37 \\
79 \cdot 02\end{array}$ & $\begin{array}{l}186 \cdot 28 \\
158 \cdot 27\end{array}$ \\
\hline
\end{tabular}

TABLE IV

PERCENTAGE CONTRIBUTION TO TOTAL DEATH RATE OF VARIOUS DISEASES (1949)

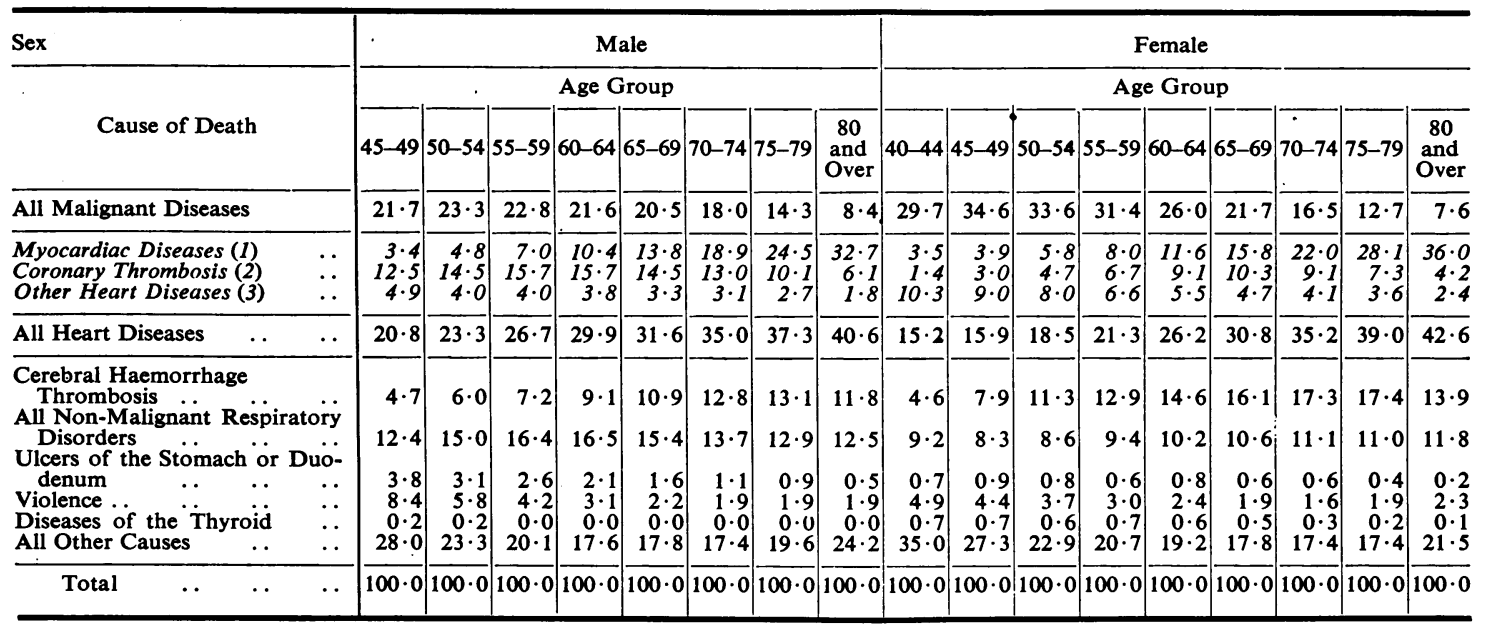

other causes of death. It is perhaps necessary here to emphasize that the expression cause of death has a special connotation in vital statistics since we cannot assign the cause and this is particularly true of the aged. Our concern is with a causal nexus, of which one component may have a special interest with regard to the course of action we intend to pursue.
The cause of death recorded in public statistics is what a physician specifies as such on a death certificate to meet the requirements of the law. The causal relevance of this may vary:

(a) as standards of differential diagnosis change,

(b) as medical educational standards change and with them what practitioners agree to specify as one and the same nosological category. 
Moreover, the procedure since 1940 gives the practitioner more scope in assigning the cause of death and the Registrar-General's staff correspondingly less scope to enforce a consistent practice.

As already stated the proper use of such statistics is to enumerate deaths assignable to one among several contributory causes rather than to any unique cause. A specification so conceived has no necessary priority over all others from the viewpoint of aetiology; but it may interest the policy maker in two ways:

(i) It may throw light on the efficacy of particular preventive and curative methods and thus indicate a target for further appropriate action.

(ii) It may clarify the need for research 'in particular directions.

A clear appreciation of what we mean by an "assigned cause of death" has a special relevance to our present theme. At the end of the 19th century infant deaths were largely associated with clearly defined communicable diseases amenable to control by preventive methods, and the annual statistics specifying the association of death with any one such disease at least ostensibly gave the policy maker a crude barometer of the success of efforts to control them. At the end of life, the picture is different, how different contemporary interest in gerontology sufficiently reminds us. For example, unless considered as a whole, deaths respectively ascribed to silocosis and to myocardiac failure in 1931 bear no intelligible relation to deaths so designated in 1955 .

Pneumoconiosis is of special interest in this context, because it has so recently become a target for legislative action. Before 1925 there was little recognition of the pneumoconioses as a nosological category, and until 1931 they evoked comparatively little attention at an administrative level. Since then various reforms intended to alleviate suffering from industrial disease have drastically altered the value of such death certificates, so that in a rough and ready way, we may distinguish four phases in the vital statistics of the period 1925-55:

(1) practitioners would ascribe nearly all deaths now ascribed to silicosis to myocardiac failure, pneumonia, or tuberculosis;

(2) practitioners might ascribe deaths to silicosis without confirmatory evidence by autopsy;

(3) the Registrar-General's office would exercise its discretion in assigning silicosis as the cause of death if this condition was mentioned on the certificate with myocardiac failure or pneumonia or tuberculosis.

(4) the Coroner's verdict based on the sworn testimony of the pathologist who performed the autopsy, is nearly always the definitive criterion.
From a biological viewpoint, we may, of course, cite silicosis on the one hand or myocardiac failure, pneumonia, or tuberculosis on the other as $a$ cause of death; but when preventive action is directed to the elimination of pneumoconiosis or when the community directly or indirectly assumes financial responsibility for its consequences, its specification as the cause of death acquires a new significance. If the community decided to take an equally urgent view of asthma as a handicap to national efficiency, the discretion of the physician in citing asthma or myocardiac failure as the cause of death would compromise the sensitivity of the statistical barometer, for the same reason.

Needless to say, myocardiac failure and heart disease in general bulk largely in the vital statistics of the older age groups whence the breakdown of deaths under myocardiac and coronary groups has been the subject of discussion by Ryle and Russell (1949). To make matters more difficult, the diagnostic classification of the General Register Office changed in 1940. Ryle and Russell considered Groups 93 (b) and (c) together with 97 (3) of the Fourth Revision of the International Classification as definitive of myocardiac diseases for the period 1931-39, but they considered Groups $93(c)$ and $(d)$ of the Fifth Revision as definitive for the period 1940-45. In what follows, the whole of Group 93 has been used. The difference which results is not small at higher ages but it is very small at lower ages. Thus for 1931, the criterion used by Ryle and Russell gives 621 deaths at age 45-49 compared with 603 on the criterion here used; for the terminal age group (80 and over), the two methods give 6,991 and 5,992 male deaths respectively. Ryle and Russell (1949, p. 388) discuss the possibility that there has been a transference from deaths previously ascribed to myocardiac disease to the more specific coronary. category, and they come to the following conclusion:

The increase in the coronary disease mortality in the post-war period is unlikely to be wholly due to transference of myocardiac deaths, because there is evidence that the coronary death-rate itself was increasing less rapidly in this period than in pre-war years-the actual death-rates at ages in 1945 being smaller than those predicted on the basis of the statistical experience relating to the period 1931-39.

It is scarcely necessary to add that similar difficulties are encountered with statistics for hypertension and nephritis. In short, it is not possible to refine further the diagnostic categories for the terminal age groups. The General Register Office has long recognized this dilemma (Registrar-General. 1949). 


\section{Breakdown by Diagnosis in the Terminal Age Groups}

A breakdown by diagnosis of the major diseases contributing to mortality from 45 years of age onwards discloses a comparatively small group of categories contributing to over 50 per cent., and in the age of groups above 50 years to well over 60 per cent. of all deaths. Tables III and IV and the Figure summarize the relevant data; they show that the changing picture reveals no clear-cut demarcation of an old-age group. On the other hand, we can broadly distinguish between three categories, which for males are clearly defined:

(a) for malignant diseases and coronary thrombosis the annual incidence falls off sharply at about 75 years of age:

(b) for myocardiac diseases, cerebral thrombosis or haemorrhage, and respiratory disorders (not illustrated) the death rate remains fairly constant from 45 years to the end of life; for myocardiac disease the incidence increases until the final age group.

(c) for violence (including accidents, etc.) the death rate rises sharply at about 65 years of age.

This three-fold distinction is also roughly applicable to females; but the fall-off for malignant diseases and for coronary thrombosis about 75 years of age is much less striking.

Sex Differences in Ageing.-The relative incidence of death from malignant disease and heart disease shows a clear sex differential. For 1949 (Table IV) the percentage contribution to all deaths for these two major causes shows the same clear shift of emphasis for each sex at a different critical age.

For males aged 45-49 the malignant diseases are slightly more important as a major cause of death; at age 50-54 the figures for the two groups of disease are equal; thereafter the heart diseases are in the ascendant, and in the final age group ( 80 years and over) they are almost five times as important.

For females the changeover comes 10 years later. Not until age 60-64 do deaths from malignant disease equal the ever-increasing deaths from heart disease. However, the heart diseases in the final age group are five and a half times as important as. the malignant diseases.

If there is to be any great improvement it must clearly come about by a decrease in the deaths now ascribed to the heart diseases.

A similar 10-year sex differential is also apparent in deaths from other causes. In Table II a small retrogression in the total death rate is first apparent for males aged 55-59 in the quinquennium 1947-51. A similar retrogression in the female death rate in the same quinquennium appears 10 years later in life, at age 65-69. Our data throw no light on how far this 10-year difference in ageing is due to social circumstances and how far to innate agencies.

\section{The Prospect for Conservation of the Aged}

For reasons stated above, the breakdown summarized in Tables III and IV is grossly and advisedly coarse, because so many of the major contributory "causes" of deaths certified as such refer to diagnostic categories of dubious value. On this understanding, and if we also grant that present indications of a slight sustained decline of mortality in the terminal age groups are not illusory, we may essay a tentative opinion on the limits that may be set to such a decline in the absence of further relevant advances of medical knowledge. Before doing so, three considerations call for comment:

(a) We have not as yet assessed how far a healthier start in life protects the individual surviving to a late age against the hazards incident thereto;

(b) Since the major sources of wastage were formerly epidemic diseases which presented a challenge less to medical research than to administrative action, a contemporary forecast for the aged is not comparable to the prospect for reduction of infant mortality when the first indications of a decline were detectable 50 years ago;

(c) Since the label attached to a very large number of deaths ascribed to "myocardiac failure" merely records the final scene of a drama the first act of which we have not witnessed, we cannot say what the course of events would have been if earlier treatment of now remediable or preventable disorders had been available at the appropriate time.

Preventive measures may accomplish an appreciable reduction of deaths classified as due to "violence", and domiciliary curative measures for pneumonia may continue to reduce the deaths directly attributable to respiratory disorders. Earlier diagnosis and treatment of peptic ulcer and the use of anticoagulants for coronary or cerebral thrombosis may conceivably accomplish a little; but our present slight knowledge of hypertension, cerebral haemorrhage and the overwhelming bulk of insidious malignant disease offers little hope of immediate improvement. We cannot therefore predict a considerable decline in mortality in the late age groups unless substantial advances in medical science occur pari-passu. Neither should we dismiss the possibility out of hand. A confident 
forecast would be justifiable only if we could assess how far protection from risks to health in early life may reduce the risk of death in the terminal years. Here the cohort method of analysis may throw some light on an otherwise obscure situation; the concluding paper in this series will disclose what further clues this method can reveal.

\section{SUMMARY}

(1) Annual age-specific death rates for the period 1931-53 show a decline for both sexes and for all age groups, with the single exception of males aged 60-64. The population estimates over this period are, however, suspect, and comparison by Life Tables based on the Census population are necessarily approximate since the final 1951 Census Population is not yet available. With these considerations in mind, there appears to be evidence of declining mortality in the terminal age groups.

(2) In the old there is rarely a single cause of death, and during this period the relevance to vital statistics of the cause stated on the death certificate has varied. A proper use of statistics based on information supplied by death certificates is to enumerate deaths assigned to causes relevant to social policy. In the later age groups, practitioners are dealing with disorders about which little is known or what is known has become known very recently. The resulting changes in classification over a period of years is illustrated by the history of the pneumoconioses, and the changes in classification of heart diseases. Therefore it is not possible to refine further the diagnostic categories for the terminal age groups.

(3) The breakdown by diagnosis of deaths of persons over 50 years of age shows that malignant diseases, heart diseases, and non-malignant respiratory diseases contribute to over 60 per cent. of all deaths. There is a sex difference in the relative importance at different ages of the two major causes, malignant diseases and heart diseases.

(4) No confident prediction of prospects for the conservation of the aged can be made from analysis of the annual rates.

Acknowledgements are due to Professor Lancelot Hogben, F.R.S., for advice and criticism.

\section{REFERENCES}

Fletcher, C. M., and Oldham, P. D. (1949). British Journal of Industrial Medicine, 6, 168

Government Actuary (G. H. Maddex) (1954). "Life Tables 1950-52", Supplement to the 99th Annual Report of the Registrar-General for Scotland, Appendix II. H.M.S.O., Edinburgh.

Registrar-General (1949). "Statistical Review of England and Wales 1940-45", Text, vol. 1, Medical, p. 77, 81 .

Ryle, J. A., and Russell,'W. T. (1949). Brit. Heart. J., 11, 370.

Taylor, W. (1954). British Journal of Preventive and Social Medicine, Taylor, 8,1 . and Dauncey, M. (1954). Ibid.. 8. 172. 